\title{
Evaluation of 'Eyelander': a video game designed to engage children and young people with homonymous visual field loss in compensatory training.
}

Jonathan Waddington ${ }^{1}$, Conor Linehan ${ }^{2}$, Kathrin Gerling ${ }^{3}$, Cathy Williams ${ }^{4}$, Leonie Robson ${ }^{5}$, Richard Ellis ${ }^{1}$, and Timothy Hodgson ${ }^{6}$.

${ }^{1}$ WESC Foundation, ${ }^{2}$ University College Cork, ${ }^{3}$ Katholieke Universeiteit Leuven, ${ }^{4}$ University of Bristol / Bristol Eye Hospital, ${ }^{5}$ United Lincolnshire Hospitals NHS Trust, ${ }^{6}$ University of Lincoln.

\begin{abstract}
Introduction: Rehabilitation can improve visual outcomes for adults with acquired homonymous visual field loss. However, it is unclear whether (re)habilitation improves visual outcomes for children because previous training schedules have been tiresome, uninteresting, and failed to keep them engaged. In this study we assessed whether children and young people with homonymous visual field loss would adhere to six weeks of unsupervised compensatory training using a specialised video game.

Methods: Participants aged between 7 and 25 with homonymous visual field loss completed table-top assessments of visual search across four site visits. Two baseline assessments separated by four weeks evaluated spontaneous improvements before training began. Participants were then given a copy of the video game to use unsupervised at home for six weeks. Two follow-up assessments separated by four weeks were then conducted to evaluate immediate and acutely maintained effects of training.
\end{abstract}


Results. 15 candidates met the inclusion-exclusion criteria, 9 participated, and 8 completed the study. Participants completed an average of 5.6 hours training unsupervised over the six weeks. Improvements on in-game metrics plateaued during week 3 of training. The time taken to find objects during table-top activities improved by an average of $24 \%$ (95\% CI [2\%, 46\%]) after training.

Discussion: The findings demonstrate that children and young people with homonymous visual field loss will engage with gamified compensatory training, and can improve visual outcomes with less time commitment than adults have required with nongamified training in previous studies. Appropriately powered, randomised controlled trials are required to evaluate the validity and generalisability of observed training effects.

Implications for practitioners: We conclude that (re)habilitation specialists can use specialist video games and gamification to engage children and young people with homonymous visual field loss in long-term unsupervised training schedules.

\section{Introduction}

One potential consequence of injury to areas of the brain that process vision is homonymous visual field loss (HVFL), in which the same region of vision is lost as seen through both eyes. In children, the most common injuries leading to HVFL are tumour (27-39\%), traumatic brain injury (19-34\%), and cerebral vascular incident (25\%) (Kedar, Zhang, Lynn, Newman, \& Biousse, 2006; Liu \& Galetta, 1997). The stereotyped pattern of visual field mapping in post-chiasmatic primary visual pathways and primary visual cortex leads to a predictable pattern of HVFL depending on the site of injury (Holmes \& Lister, 1916; Horton \& Hoyt, 1991; Inouye, 2000). An injury that completely interrupts the optic radiations in the right cerebral hemisphere typically results in complete loss of the left half of the visual field in both eyes (i.e. left homonymous hemianopia). This pattern of visual field mapping is 
consistent to the degree that HVFL is congruent (identical between the two eyes) in approximately 84\% of cases (Kedar, Zhang, Lynn, Newman, \& Biousse, 2007). HVFL that respects the vertical midline such as left or right sided hemianopia affects an estimated 11$22 \%$ of children with cerebral vision impairment, but altitudinal or peripheral visual field loss may affect as many as 49\% (Bosch, Boonstra, Willemsen, Cremers, \& de Vries, 2014; Huo, Burden, Hoyt, \& Good, 1999).

There is an acute period of approximately three months after brain injury during which 8-20\% of adults with HVFL may spontaneously recover their entire visual field, and as many as 50-60\% may partly recover (Gray et al., 1989; Rowe et al., 2013; Zhang, Kedar, Lynn, Newman, \& Biousse, 2006). Evidence for spontaneous recovery of HVFL in children is scarce and typically based on case studies. However, it is estimated that $50-84 \%$ of children with cerebral vision impairment will partially recover or develop some functional vision over time, with a poorer prognosis for those patients that have injury to the periventricular white matter (Casteels et al., 1997; Hoyt, 2003; Huo, Burden, Hoyt, \& Good, 1999; Roland, Jan, Hill, \& Wong, 1986). Rehabilitation strategies for adult stroke patients with HVFL have been investigated over the last two decades with varying degrees of effectiveness at improving functional vision (Bouwmeester, Heutink, \& Lucas, 2007; Kerkhoff, 2000; Lane, Smith, \& Schenk, 2008; Pambakian, Currie, \& Kennard, 2005; Pelak, Dubin, \& Whitney, 2007; Pollock et al., 2011; Trauzettel-Klosinski, 2011). These strategies are typically categorised into one of three groups: compensation, restitution, or supplementation. Compensation aims to adapt behaviour to compensate for lost visual function, restitution aims to restore visual function, and supplementation involves using optical aids such as prisms or sensory substitution devices to improve functional vision.

Compensatory approaches include structured training of large saccades into the area of HVFL, or training of scanning patterns using visual search tasks or scrolling text. 
Restitution therapy involves repetitively targeting high contrast visual stimuli within or on the border of the area of HVFL. There is evidence to favour the use of compensatory training rather than restitution therapy in adults (Pollock et al., 2011). Improvements in visual outcomes after compensatory training are not significantly different between younger (20-34 years old) and older (70-84 years old) adults (Schuett \& Zihl, 2013). There is some limited evidence that restitution therapy may be more effective for children than adults, but very few studies have investigated the effectiveness of compensatory training in children (Waddington \& Hodgson, 2017; Werth \& Moehrenschlager, 1999; Werth \& Seelos, 2005). A particular problem is that compensatory training requires weeks of adherence to daily routines involving tiresome and uninteresting tasks.

The process of designing and developing a therapeutic video game called 'Eyelander' to motivate children with HVFL to undertake compensatory training has been previously reported (Waddington, Linehan, Gerling, Hicks, \& Hodgson, 2015). In this study we assessed 'Eyelander' as a therapeutic intervention to determine whether children and young people with HVFL would engage with the training unsupervised at home.

\section{Methods}

Participants

This study received ethical approval from the University of Lincoln School Of Psychology Research Ethics Committee as well as the United Kingdom (UK) National Research Ethics Service Committee North East - Newcastle \& North Tyneside 1.

We opened 4 participant identification centres in different regions within the UK, including research sites from both the health and education sectors. Professionals including 
ophthalmologists, orthoptists, research nurses, and qualified teachers of students with visual impairments identified candidate participants. The initial approach to candidates was via information booklet to the parent or young adult with a reply slip to opt in. We used inclusion criteria for age (7-25 years) and the suspected presence of HVFL ranging in severity from quadrantanopia or sectoral defect to complete hemianopia. We excluded participants with profound physical and/or cognitive impairments, but included participants with additional mild or moderate impairments if they were able to access the software with or without the aid of access technology. We identified 15 candidates, and 9 participants ( 6 female; median age $=$ 10.3 years, and age range $=7-21$ years) gave informed consent or assented with parental consent.

Participants who were unable to provide confirmation of HVFL from their doctor or eye care specialist were asked to perform a central 24-2 threshold test using a Humphrey visual field analyser and to visit an ophthalmologist and orthoptist for a vision assessment to rule out retinal causes of visual field loss. Four participants presented with right-sided homonymous hemianopia, 2 participants presented with left-sided homonymous hemianopia, 1 participant presented with a right-sided sectoral defect, and 2 participants presented with incongruent lower altitudinal visual field loss. All participants were in the chronic stages of recovery, at least 3 months after symptoms of vision impairment had been reported. All participants completed a line bisection test and demonstrated no obvious evidence of spatial neglect on this test. Eight participants demonstrated a degree of physical impairment that limited either their mobility, or their ability to use a mouse and keyboard, or both. Cognitive impairment was not formally assessed although anecdotal evidence indicated a range of cognitive abilities from neurotypical to moderate learning difficulties. All participants were able to communicate, understand, and follow instructions with age-appropriate language. 
One participant (male, 9 years old, with left-sided hemianopia) dropped out of the study after one month due to a change in care support at home. We therefore present the results of our investigation with one group of 8 participants.

Training paradigm and in-game data collection

Participants were asked to undertake unsupervised compensatory training in their home using the video game 'Eyelander' for a period of approximately six weeks. We did not control the training environment, viewing distance, or screen size of the presented software. The software was provided as an executable program on a USB flash drive and was playable on a home computer or laptop running a Windows operating system (XP, Vista, 7, 8).

The design and development of the game has been documented elsewhere (Waddington et al., 2015) so here we give only a brief summary of the training paradigm. The premise of the training was a modification of a visual search task used in previous studies to rehabilitate adult stroke patients with HVFL (Pambakian, Mannan, Hodgson, \& Kennard, 2004). Search trials could be of the single feature or conjunction type. Feature searches comprised sets of target objects amongst distractor objects that differed by a single feature (shape, colour, rotation, size, 'flashing on/off'). Conjunction searches included two types of distractors each differing from the target by one of two possible features. Participants were asked to move the cursor to point at the target and press the left mouse button or pressed the right button if the target was absent. Feedback was given following response in the form of high or low tones and a variety of visual "particle effects" (such as the shape object exploding). Participants completed search trials to progress through 12 levels of game narrative, passing through various obstacles to escape from a mysterious desert island. Sets 14 contained 1, 2, 4, and 8 feature search trials respectively. Sets 5-12 each contained 12 
search trials with a $10 \%$ chance that any trial would be a target absent trial. At the start of sets 5-12 there was a chance (10\%-80\% respectively) that any given trial would be a conjunction search. Within each set the probability of a conjunction search increased by $10 \%$ for every successful trial completed and reset to zero if three errors occurred in succession.

All participants were given twenty minutes of demonstration and training time with one of the researchers when they first received a copy of the game. The researcher manually calibrated the difficulty settings so that the participant could access the game. Additional instructions were embedded into the game including an optional tutorial. The number of trials completed, percentage of targets found, response time, and target location were logged on the computer after each session of play. These data were analysed offline after the training period to retrospectively assess compliance and progress with the training.

Table-top tests of visual search ability

We measured participant performance on table-top search tasks to assess transference of visual search skills learned playing the video game to other activities. Five timed tasks were devised by the authors and validated with a group of 122 participants without vision impairment between the ages of 3-11 years, enabling us to determine age adjusted expected performance for the tests. Participants were a convenience sample of children who were attending a week-long public engagement event at the University of Lincoln and had no selfreported neurological conditions or vision impairment. Each participant completed five different table-top tasks once.

Each task involved searching for a number of specified target objects among distracting objects on a table covered with a black tablecloth. Task 1 included 3 sets of 8 
coloured blocks (red, blue, yellow), task 2 included 4 sets of 6 geometric blocks (squares, circles, triangles, rectangles), task 3 included 3 sets of 8 UK currency coins (1p, 2p, 5p), task 4 used 26 uppercase letters of the English alphabet (5 randomly selected letters were chosen as targets), and task 5 utlised 4 sets of 2 coloured compact disc (CD) cases (red, blue, green, yellow). Test order was randomly allocated prior to each assessment. For tasks 1-4 the objects were first placed by the experiment in a random distribution around an A3 white card in front of the participant. The card was then turned over to reveal a depiction of which target objects should be searched for with a verbal prompt. Participants indicated they had found the correct objects by picking them up and placing them on the card. Task 5 was prepared by arranging CD cases at both ends of a $0.8 \mathrm{~m}$ long CD rack before asking the participant to find the target colour cases. The time taken to complete each task and the number of target objects found were recorded.

We calculated a simple linear regression to predict log transformed visual response times based on the log transformed age of participants. A significant regression equation was found $\left(F(1,107)=202, p<2.0 \times 10^{-26}\right)$, with an adjusted $R^{2}$ of 0.651 . The participants' predicted visual response times were equal to $70.0 \mathrm{age}^{-1.68}$ seconds when age was measured in years. Based on this regression analysis we found the expected value and 95\% expectation interval (domain between the $2.5^{\text {th }}$ and $97.5^{\text {th }}$ percentiles) of visual response time for our control participants to be $1.38 \mathrm{~s},[0.67 \mathrm{~s}, 2.86 \mathrm{~s}]$ at 10.3 years old (the median age of our participants with HVFL). We used these as reference values and will present a more detailed analysis of the data collected from our control participants in a separate paper.

Participants with HVFL performed the table-top tests of visual search ability in random order at each of four site visits in an intermittent time series design. Two site visits were performed before ( $\mathrm{t} 1$ and $\mathrm{t} 2$ ) and two site visits after ( $\mathrm{t} 3$ and $\mathrm{t} 4$ ) the period playing the video game. Site visits were scheduled such that the pre-training period (t1 to t2) was 3-5 
weeks, the training period ( $\mathrm{t} 2$ to $\mathrm{t} 3$ ) was $5-7$ weeks, and the post-training period ( $\mathrm{t} 3$ to $\mathrm{t} 4$ ) was 3-5 weeks, depending on when participants and parents were able to attend. Assessors were not blinded to the number of previous site visits but were blinded to the amount of times the game had been played and number of search trials completed. The distribution of visual response times recorded for these tasks was highly positively skewed such that parametric tests on logarithmic transformation of visual response time data and ranked visual response time were used in the statistical analysis.

We also assessed a participant reported outcome measure of visual ability using the Cardiff Visual Ability Questionnaire for Children (CVAQC), and a measure of health-related quality of life using the Impact of Vision Impairment for Children (IVI_C) questionnaire. Both questionnaires have been validated for use with vision impaired children and give a unidimensional score (Cochrane, Marella, Keeffe, \& Lamoureux, 2011; Khadka, Ryan, Margrain, Court, \& Woodhouse, 2010). Both questionnaires were completed verbally at site visits before (t2) and after (t3) training with the video game.

\section{Results}

Error rates and response times within the video game

Participants on average played the game on 16.1 separate days (95\% CI [8.7 days, 23.6 days]) and completed 1676 visual search trials (95\% CI [850 trials, 2540 trials]) over the total training period. There was some evidence of disengagement as they played the game on 4.1 days during week 1 (95\% CI [2.5 days, 5.8 days]), 3.0 days during week 2 (95\% CI [1.1 days, 4.9 days]), and approximately 2 days during each week after that (fig.1). 
The percentage of targets correctly found (response accuracy) started at a high score on average of $87 \%$ on the first day (95\% CI [76\%, 98\%]). Response accuracy increased slightly over the first two weeks but reached a peak of 94\% during week 3 (95\% CI [89\%, $100 \%]$ ) and saturated in the weeks after that (fig.2A). We performed a Paired Samples T test to compare the weekly average response accuracy to targets in affected quadrants with the weekly average response accuracy to targets in quadrants not affected, and found a significant difference of just $-2.6 \%$, 95\% CI [-0.8\%, $-4.4 \%](t=3.54, d f=6, p=0.012)$. This difference in response accuracy between quadrants affected and not affected appeared relatively minor. However, this may be due to response accuracy to targets in affected quadrants improving over the training period such that the response accuracy became more similar across all quadrants over time (fig.2A), obscuring the initial difference.

We analysed the statistics for the log transformed response time data, and converted means and confidence intervals back into the time domain to simplify interpretation. The response time to correctly select visual targets started at an average of 3.21s on the first day (95\% CI [2.7s, 3.8s]). The average response time decreased slightly over the first two weeks but reached a nadir of 2.78s during week 3 (95\% CI [2.3s, 3.4s]) and began to increase rapidly in the weeks after that (fig.2B). We performed a Paired Samples T test to compare the weekly average response time to targets in affected quadrants with the weekly average response time to targets in quadrants not affected, and found a significant difference of 0.11 $\log$ units, 95\% CI [0.06 log units, $0.17 \log$ units] $(t=5.01, d f=6, p=0.002)$. As small differences in the natural log of a variable can be interpreted as percentage changes in the variable itself this indicated that visual response times were on average $11 \%$ slower to targets in affected quadrants when compared with visual response times to targets in quadrants not affected. Both learning curves were dominated by a rapid slowing in response times in the 
latter half of the training period (fig.2B), which appeared to indicate diminishing returns on continued training.

Table-top tests of visual search ability

None of the participants missed more than one target object during table-top tests. We therefore do not present the response accuracy data here. Visual response times are known to be significantly positively skewed and non-Gaussian. We therefore present both the logtransformed visual response times and the ranked visual response times ( 1 = fastest, $4=$ slowest) in Figures 3 and 4 respectively for comparison.

The mean values of log-transformed visual response time were $4.37 \mathrm{~s}$ at $\mathrm{t} 1,4.69 \mathrm{~s}$ at t2, 3.68s at t3, and 3.56s at t4 (fig.3A). These average values were significantly slower than the expected value estimated from our control participants of $1.38 \mathrm{~s}\left(97.5^{\text {th }}\right.$ percentile $\left.=2.86 \mathrm{~s}\right)$ for a child of 10.3 years in age. We performed a Repeated Contrast Analysis of Variance on the log transformed visual response time data and found a marginal difference between the mean values at $\mathrm{t} 2$ and $\mathrm{t} 3(F=4.56, d f=1, p=0.070)$, no difference between values at $\mathrm{t} 1$ and $\mathrm{t} 2$ ( $F=0.18, d f=1, p=0.69)$, and no difference between values at $\mathrm{t} 3$ and $\mathrm{t} 4(F=0.22$, $d f=1, p=0.66)$.

When we compared visual response times averaged over both pre- and post-training assessments with a Paired Samples T Test we found that there was a statistically significant improvement ( $t=2.56, d f=7, p=0.037)$. On average visual response times decreased after training by $0.24 \log$ units (95\% CI [0.02 log units, 0.46 log units]) from 4.63s (95\% CI [2.90s, 7.38s]) to 3.64s (95\% CI [2.34s, 5.65s]) (fig.3B). This indicated that visual response 
times during table-top tests of visual search ability had improved after training on average by $24 \%(95 \%$ CI $[2 \%, 46 \%]$, SD $=27 \%)$.

The mean ranks of the visual response time data were 3.38 at t1, 3.00 at t2, 1.75 at t3, and 1.88 at $\mathrm{t} 4$, indicating that visual response times were ranked fastest at $\mathrm{t} 3$ and slowest at $\mathrm{t} 1$ (fig.4). We performed a Repeated Measures Analysis of Variance on the ranked visual response time data and found a significant main effect of the time of assessment on ranks $(F=4.55, d f=3, p=0.013)$. We performed a Repeated Contrast Analysis of Variance on the ranked data and found a significant statistical difference between ranks at $\mathrm{t} 2$ and $\mathrm{t} 3$ ( $F=$ 11.7, $d f=1, p=0.011)$ but not between ranks at t1 and t2 $(F=0.30, d f=1, p=0.60)$ or ranks at t3 and t4 $(F=0.080, d f=1, p=0.79)$. This indicated that visual response times during the table-top tests were significantly faster after the training period, and that no significant differences were observed after the pre- or post-training periods.

We investigated confounding variables that we had not controlled in the experimental design such as age and the number of trials completed during training to determine whether there were any correlations between these variables and the effectiveness of the training. Surprisingly, we did not observe any dependency on age but this could be due to the small number of participants. However, we found a strong correlation between the number of trials attempted during training and the difference in log-transformed visual response times between $\mathrm{t} 3$ and $\mathrm{t} 4\left(F(1,6)=100, p<5.8 \times 10^{-5}\right.$, adjusted $\left.R^{2}=0.934\right)$. This correlation was positive meaning that completing more search trials was related to a greater increase in response time between $\mathrm{t} 3$ and $\mathrm{t} 4$. In other words, those participants who played the game the most were those least likely to maintain their improvements on the table-top tests of visual search ability. 


\section{Participant reported outcome measures}

Data from the CVAQC were recorded for all 8 participants who completed the training. However, one participant did not complete the IVI_C questionnaire at their first visit because they did not feel the questions were appropriate for their age and circumstances. On average participants were unable or did not answer 23\% (95\% CI [15\%, 32\%]) of questions from each CVAQC and did not answer 6\% (95\% CI [1\%, 11\%]) of questions from each IVI_C questionnaire. Missing data within each questionnaire were interpolated using the mean value taken across the remaining data.

Participants reported improvements in visual ability that were not significantly different to zero as an average increase in the CVAQC score of +3.6\% (95\% CI [-1.5\%, $+8.7 \%], \mathrm{SD}=6.1 \%)$ from a baseline measure of $63 \%(95 \%$ CI $[55 \%, 71 \%])$. Participants reported improvements in quality of life that were not significantly different to zero as an average increase in the IVI_C score of $+3.2 \%(95 \%$ CI $[-1.8 \%,+8.2 \%]$, SD $=5.4 \%)$ from a baseline measure of 69\% (95\% CI [62\%, 75\%]).

\section{Discussion}

In this study children and young people with HVFL successfully engaged in unsupervised compensatory training over a six week training period using a video game specifically designed for this purpose. We had 60\% uptake from eligible candidates and an 11\% drop-out rate (1 participant) due to reduced care support at home, indicating 53\% compliance with the training. We recommended that participants play the game for 5 sessions during each week of training and complete all 111 visual search trials during each session. The participants that attended all our assessments completed 16.1 sessions of training and attempted approximately 
1676 visual search trials on average. This equated to completing $52 \%$ of the recommended number of search trials, committing 55 minutes to training each week on average.

Participants showed a significant improvement in visual search skills after training that transferred to table-top tests, equating to a $25 \%$ improvement in response times. However, we did not observe an improvement in patient reported quality of life.

It is typically thought that younger people have an increased capacity for neuroplastic changes and recovery from brain injury (Johnston, 2009; Kennard, 1936; Teuber, 1974). We did not observe any relationship between the age and the effects of training in the current study. Previous work has found no significant difference in outcome measures between younger adults and older adults after compensatory training (Schuett \& Zihl, 2013). We based our design and training protocol on a previous study of adult stroke patients with HVFL, in which participants also performed table-top tests 25\% faster after training (Pambakian et al., 2004). The similarity between the previous and current effect size suggests that compensatory search training may be equally effect in children and adults with HVFL.

It is interesting to compare the level of compliance in this study with three previous in adults using non-gamified training (Aimola et al., 2014; de Haan, Melis-Dankers, Brouwer, Tucha, \& Heutink, 2015; Mödden et al., 2012). These studies indicated a 60\% compliance rate, and a commitment to training of 2.35 hours per week (range: 1.85 - 2.69 hours per week) for 7.4 weeks (range: 3 - 10 weeks) on average. Whereas the participants in the current study committed to 0.9 hours per week for 6.25 weeks on average. The finding that children and young people make comparable improvements in visual outcomes after only completing a fraction of the training that adults have in previous studies might indicate that children and young people beneift from training more quickly than adults. Alternatively, differences in learning speed may be due to the benefits of a gamified training protocol compared to standard training protocols. 
The improvements we observed from participants on in-game metrics appeared to plateau during the third week of training despite in-game dynamic difficulty adjustment. Additionally, we found a negative withdrawal effect at the final follow-up assessment from those participants who had attempted the most trials during the training period. These results appear to indicate that excessive commitment to training does not necessarily translate to additional functional improvements, and may even be detrimental to maintaining improvements in the long-term. Further research is required to determine what the optimal schedule for rehabilitative training is.

This study has a number of limitations, the most important of which are the relatively small number of participants involved and the lack of a control group that underwent a placebo intervention. A larger, powered, randomised controlled trial would be required to confirm the validity and generalisability of the observed training effects. This is especially important as the variability in test performance between our participants was large compared to the spread of their averages. We should therefore be careful about how much value we place on the interpretation of a statistically significant difference. However, the effect size found with this small group of children and young people is similar to that found in previous controlled studies, which included slightly larger sample sizes of adults with HVFL participating in compensatory training (Lane, Smith, Ellison, \& Schenk, 2010; Roth et al., 2009).

There is evidence to suggest that intermittent and variable positive feedback schedules that are common in gaming can enhance the effectiveness of learning through maximising surprise (Linehan, Kirman, Lawson, \& Chan, 2011; Wills, 2011; Wills, Lavric, Croft, \& Hodgson, 2007). As such, an appropriately controlled trial could also be used to assess whether gamified training can improve visual outcomes more than non-gamified training, in adults as well as children. Two themes from participants for improving the engaging qualities 
of the game were the creation of additional narrative content and making the software available on a mobile platform. Both of these suggestions require further consideration. Narrative content is typically expensive and time consuming to produce, and mobile platforms may not have a large enough screen size to promote beneficial scanning strategies.

Another limitation is that we did not track eye or head movements in this study so the mechanism of effect remains somewhat unclear. A previous study has demonstrated that training with psychophysics attention trials can improve visual search times for young people with low vision due to ocular conditions (Nyquist, Lappin, Zhang, \& Tadin, 2016). However, whether improvements in visual outcomes for young people with ocular conditions and young people with HVFL are mediated by a common mechanism of effect requires further investigation. We did not find any evidence of a relationship between spatial neglect and training effects and none of our participants demonstrated any signs of spatial neglect on a line bisection test. Given the nature of our training protocol and the results of previous studies including adult stroke patients with HVFL discussed in our introduction, we suspect improvements were mediated by a combination of improved visual attention and more systematic scanning strategies (conscious, subconscious, or both), not changes in the border of the visual field itself.

It should be noted that whilst the improvements in visual search performance were not found to be associated with changes in quality of life outcomes as assessed by CVAQC and IVI_C questionnaires (Cochrane et al., 2011; Khadka et al., 2010), the large proportion of missing responses make this finding difficult to interpret. This was because many of the questions lacked relevance to participants. We recommend that future research should focus on developing validated tools specifically targeted at measuring the quality of life of children and young people with neurological visual impairments. 
We conclude that children and young people with HVFL can improve their functional vision unsupervised using gamified compensatory training. We recommend that (re)habilitation specialists consider signposting children and young people with HVFL as early as appropriate to such software for use in between scheduled teaching, training, and therapy sessions. Improvements during training with 'Eyelander' translated to a $24 \%$ improvement in speed during table-top tests of visual search ability. This improvement is comparable to results from previous studies in which adult stroke patients with HVFL completed non-gamified training. However, the children and young people in this study only had to commit to training for a fraction of the time adults did and there is some evidence that over-commitment to training was not beneficial. As such, practitioners may wish to recommend that children and young people with HVFL only commit to an hour of training each week for 4-5 weeks outside of their usual teaching, training, and therapy sessions. Whether these training effects are generalisable, and whether this difference in learning speed is due to the age of the participants or the use of gamification requires further investigation.

\section{Acknowledgement}

We acknowledge the time contributed by all participants and the support of the National Institute of Health Research Clinical Research Network (NIHR CRN). We acknowledge the time contributed to help identify participants for the study by Rebekka Coles, Katrina Cooke, Paul Tesha, Pam King, and Sally Malloy. We also acknowledge the time and work contributed to the design and development of Eyelander by Kieran Hicks, Robert Banks, Kitiara Waddington, Alex Norgate, Caroline Burkie, and the participant co-designers. 
This work was supported by funding from the WESC Foundation, the Technology Strategy Board (now Innovate UK), and the Medical Research Council of the United Kingdom, as part of a Knowledge Transfer Partnership (Ref: KTP008989).

\section{References}

Aimola, L., Lane, A., Smith, D., Kerkhoff, G., Ford, G., \& Schenk, T. (2014). Efficacy and feasibility of home-based training for individuals with homonymous visual field defects. Neurorehabil Neural Repair, 28(3), 207-218.

Bosch, D., Boonstra, F., Willemsen, M., Cremers, F., \& de Vries, B. (2014). Low vision due to cerebral visual impairment: differentiating between acquired and genetic causes. BMC Ophthalmol, 14, 59. doi: 10.1186/1471-2415-14-59.

Bouwmeester, L., Heutink, J., \& Lucas, C. (2007). The effect of visual training for patients with visual field defects due to brain damage: a systematic review. J Neurol Neurosurg Psychiatry, 78(6), 555-564.

Casteels, I., Demaerel, P., Spileers, W., Lagae, L., Missotten, L., \& Casaer, P. (1997). Cortical visual impairment following perinatal hypoxia: Clinicoradiologic correlation using magnetic resonance imaging. J Pediatr Ophthalmol Strabismus, 34(5), 297-305.

Cochrane, G., Marella, M., Keeffe, J., \& Lamourex, E. (2011). The impact of vision impairment for children (IVI_C): Validation of a vision-specific pediatric quality-oflife questionnaire using Rasch analysis. Invest Ophthalmol Vis Sci, 52(3), 1632-1640. 
Gray, C., French, J., Bates, D., Cartlidge, N., Venables, G., \& James, O. (1989). Recovery of visual fields in acute stroke: Homonymous hemianopia associated with adverse prognosis. Age Ageing, 18(6), 419-421.

de Haan, G., Melis-Dankers, B., Brouwer, W., Tucha, O., \& Heutink, J. (2015). The effects of compensatory scanning training on mobility in patients with homonymous visual field defects: A randomized controlled trial. PLoS One, 10(8), e0134459. doi: 10.1371/journal.pone.0134459.

Holmes, G., \& Lister, W. (1916). Disturbances of vision from cerebral lesions, with special reference to the cortical representation of the macula. Brain, 39(1-2), 34-73.

Horton, J., \& Hoyt, W. (1991). The representation of the visual field in human striate cortex. A revision of the classic Holmes map. Arch Ophthalmol, 109(6), 816-824.

Hoyt, C. (2003). Visual function in the brain-damaged child. Eye (Lond), 17(3), 369-384.

Huo, R., Burden, S., Hoyt, C., \& Good, W. (1999). Chronic cortical visual impairment in children: Aetiology, prognosis, and associated neurological deficits. Br J Ophthalmol, 83(6), 670-675.

Inouye, T. (2000). Visual disturbances following gunshot wounds of the cortical visual area: based on observations of the wounded in the recent Japanese wars: 1900, 1904-05. Brain, 123(suppl), 1-101.

Johnston, M. (2009). Plasticity in the developing brain: Implications for rehabilitation. Dev Disabil Res Rev, 15(2), 94-101.

Kedar, S., Zhang, X., Lynn, M., Newman, N., \& Biousse, V. (2006). Pediatric homonymous hemianopia. J AAPOS, 10(3), 249-252. 
Kedar, S., Zhang, X., Lynn, M., Newman, N., \& Biousse, V. (2007). Congruency in homonymous hemianopia. Am J Ophthalmol, 143(5), 772-780.

Kennard, M. (1936). Age and other factors in motor recovery from precentral lesions in monkeys. Am J Physiol, 115, 138-146.

Kerkhoff, G. (2000). Neurovisual rehabilitation: Recent developments and future directions. $J$ Neurol Neurosurg Psychiatry, 68(6), 691-706.

Khadka, J., Ryan, B., Margrain, T., Court, H., \& Woodhouse, J. (2010). Development of the 25-item Cardiff visual ability questionnaire for children (CVAQC). Br J Ophthalmol, 94(6), 730-735.

Lane, A., Smith, D., Ellison, A., \& Schenk, T. (2010). Visual exploration training is no better than attention training for treating hemianopia. Brain, 133(6), 1717-1728.

Lane, A., Smith, D., \& Schenk, T. (2008). Clinical treatment options for patients with homonymous visual field defects. Clin Ophthalmol, 2(1), 93-102.

Linehan, C., Kirman, B., Lawson, S., \& Chan, G. (2011, May). Practical, appropriate, empirically-validated guidelines for designing educational games. In: CHI '11 Proceedings of the SIGCHI Conference on Human Factors in Computing Systems. Paper presented at CHI 2011: The ACM CHI Conference on Human Factors in Computing Systems, Vancouver BC Canada (pp.1979-1988). New York NY USA: ACM Press.

Liu, G., \& Galetta, S. (1997). Homonymous hemifield loss in childhood. Neurology, 49(6), 1748-1749. 
Mödden, C., Behrens, M., Damke, I., Eilers, N., Kastrup, A., \& Hildebrandt, H. (2012). A randomized controlled trial comparing 2 interventions for visual field loss with standard occupational therapy during inpatient stroke rehabilitation. Neurorehabil Neural Repair, 26(5), 463-469.

Nyquist, J., Lappin, J., Zhang, R., \& Tadin, D. (2016, Nov). Perceptual training yields rapid improvements in visually impaired youth. Sci Rep, 6, 37431. doi: 10.1038/srep37431.

Pambakian, A., Currie, J., \& Kennard, C. (2005). Rehabilitation strategies for patients with homonymous visual field defects. J Neuroophthalmol, 25(2), 136-142.

Pambakian, A., Mannan, S., Hodgson, T., \& Kennard, C. (2004). Saccadic visual search training: A treatment for patients with homonymous hemianopia. J Neurol Neurosurg Psychiatry, 75(10), 1443-1448.

Pelak, V., Dubin, M., \& Whitney, E. (2007). Homonymous hemianopia: A critical analysis of optical devices, compensatory training, and NovaVision. Curr Treat Options Neurol, 9(1), 41-47.

Pollock, A., Hazelton, C., Henderson, C., et al. (2011). Interventions for visual field defects in patients with stroke. Cochrane Database Syst Rev, 2011(10), CD008388. doi: 10.1002/14651858.CD008388.pub2.

Roland, E., Jan, J., Hill, A., \& Wong, P. (1986). Cortical visual impairment following birth asphyxia. Pediatr Neurol, 2(3), 133-137.

Roth, T., Sokolov, A., Messias, A., Roth, P., Weller, M., \& Trauzettel-Klosinski, S. (2009). Comparing explorative saccade and flicker training in hemianopia: A randomized controlled study. Neurology, 72(4), 324-331. 
Rowe, F., Wright, D., Brand, D., et al. (2013). A prospective profile of visual field loss following stroke: Prevalence, type, rehabilitation, and outcome. Biomed Res Int, 2013, 719096. doi: 10.1155/2013/719096.

Schuett, S., \& Zihl, J. (2013). Does age matter? Age and rehabilitation of visual field disorders after brain injury. Cortex, 49(4), 1001-1012.

Teuber, H. (1974). Functional recovery after lesions of the nervous system. II. Recovery of function after lesions of the central nervous system: history and prospects. Neurosci Res Program Bull, 12(2), 197-211.

Trauzettel-Klosinski, S. (2011). Current methods of visual rehabilitation. Dtsch Ärztebl Int, 108(51-52), 871-878.

Waddington, J., \& Hodgson, T. (2017). Review of rehabilitation and habilitation strategies for children and young people with homonymous visual field loss caused by cerebral vision impairment. British Journal of Visual Impairment, 35(3), 197-210.

Waddington, J., Linehan, C., Gerling, K., Hicks, K., \& Hodgson, T. (2015, April). Participatory design of therapeutic video games for young people with neurological vision impairment. In: CHI '15 Proceedings of the $33^{\text {rd }}$ Annual ACM Conference on Human Factors in Computing Systems. Paper presented at CHI 2015: The ACM CHI Conference on Human Factors in Computing Systems, Seoul Republic of Korea (pp.3533-3542). New York NY USA: ACM Press.

Werth, R., \& Moehrenschlager, M. (1999). The development of visual functions in cerebrally blind children during a systematic visual field training. Restor Neurol Neurosci, 15(23), 229-241. 
Werth, R., \& Seelos, K. (2005). Restitution of visual functions in cerebrally blind children. Neuropsychologia, 43(14), 2011-2023.

Wills, A. (2011). Learning from errors: Perspectives from basic research. In: E. Wuttke \& J. Seifried (Eds.), Learning from Errors at School and at Work (pp.143-153). Leverkusen Germany: Verlag Barbara Budrich.

Wills, A., Lavric, A., Croft, G., \& Hodgson, T. (2007). Predictive learning, prediction errors and attention: Evidence from event-related potentials and eye-tracking. $J$ Cogn Neurosci, 19(5), 843-854.

Zhang, X., Kedar, S., Lynn, M., Newman, N., \& Biousse, V. (2006). Natural history of homonymous hemianopia. Neurology, 66(6), 901-905. 


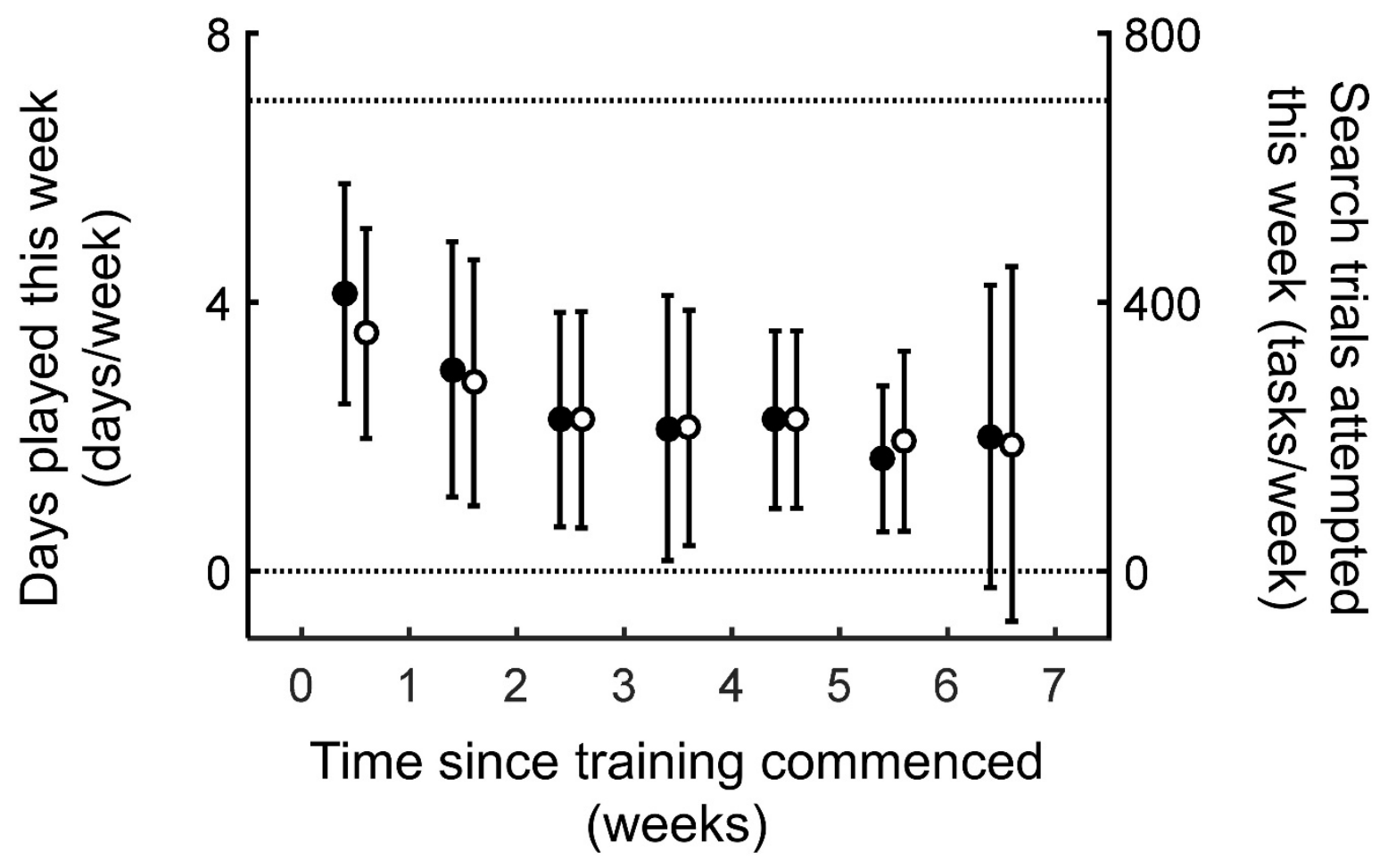

Figure 1: Changes in levels of engagement with the game over the training period.

Mean number of days played (filled circles) and search trials attempted (empty circles) for the participants as a group, recorded each week during the training period. Error bars $=95 \%$ CI. Dotted lines = minimum and maximum number of days played per week, for reference. 
A

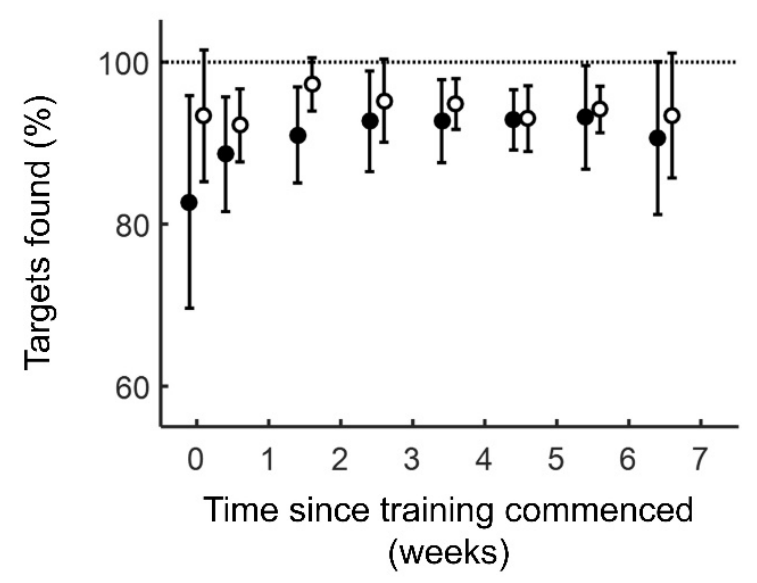

B

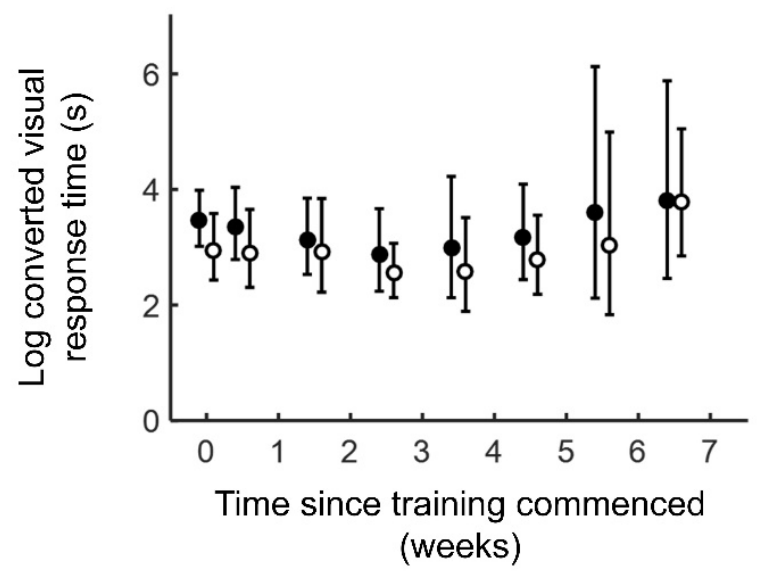

Figure 2: Changes in response accuracy and log-transformed visual response times within the game over the training period.

Mean (A) percentage of targets found and (B) log-transformed visual response times to find targets that were displayed in affected quadrants (filled circles) and quadrants not affected (empty circles) for the participants as a group, recorded each week during the training period and on the first day. Error bars $=95 \%$ CI. Dotted line $=$ maximum percentage of targets found, for reference. Note that log-transformed visual response time data and error bars are displayed on a raw time axis to simplify interpretation. 

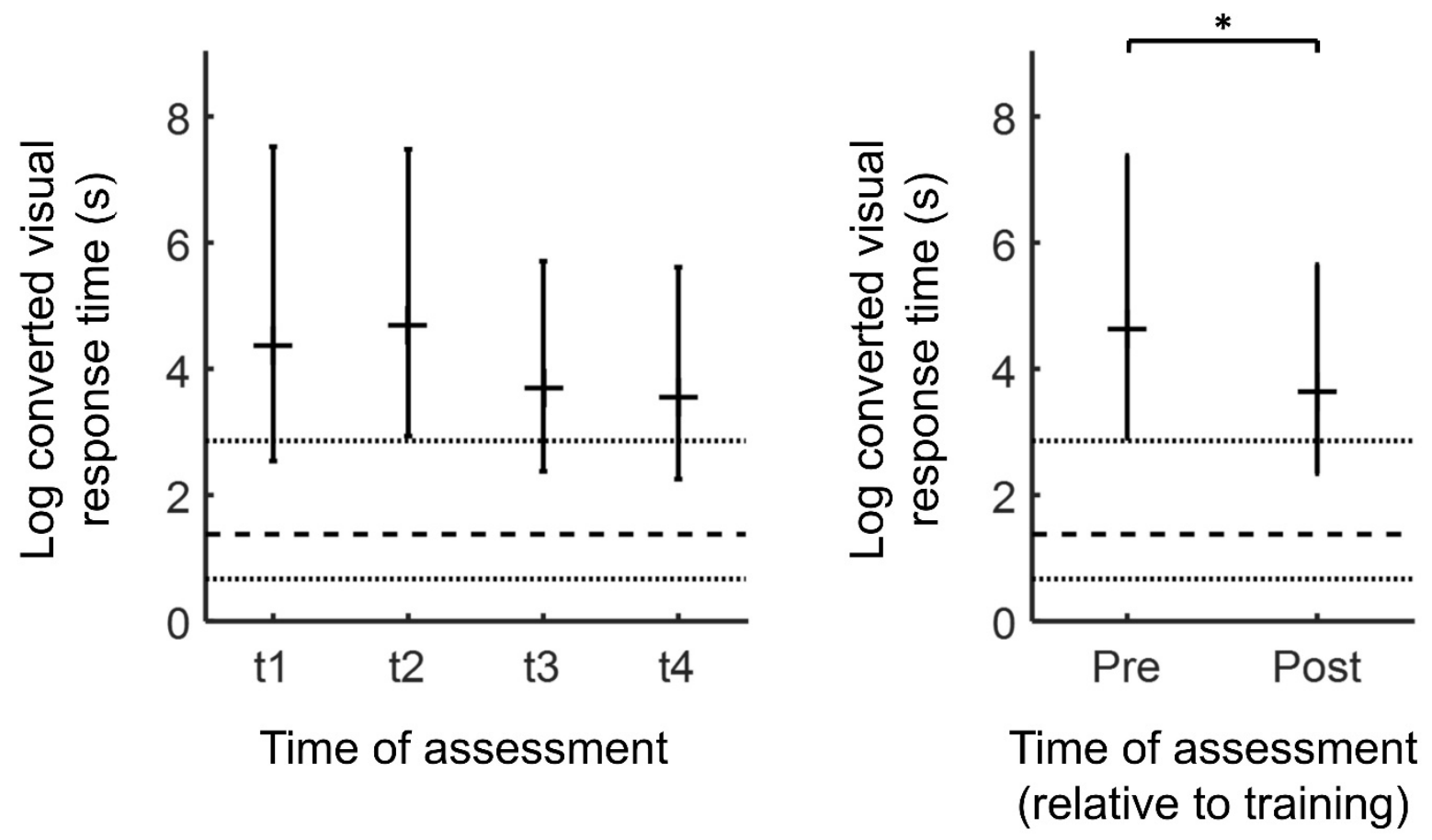

Figure 3: Changes in log-transformed visual response times during table-top tests of visual search ability before and after training.

Mean log-transformed visual response times during table-top tests of visual search ability for the participants as a group (A) at all four assessments, and (B) averaged over the two pretraining assessments and two post-training assessments. Error bars $=95 \%$ CI. Dashed line $=$ estimated mean visual response time for a control participant with no vision impairment at 10.3 years old, for reference. Dotted lines $=$ estimated $2.5^{\text {th }}$ and $97.5^{\text {th }}$ percentiles of visual response time for a control participant with no vision impairment at 10.3 years old, for reference. $*=$ significant difference, $\mathrm{p}<0.05$. Note that log-transformed visual response times and error bars are displayed on a raw time axis to simplify interpretation. 


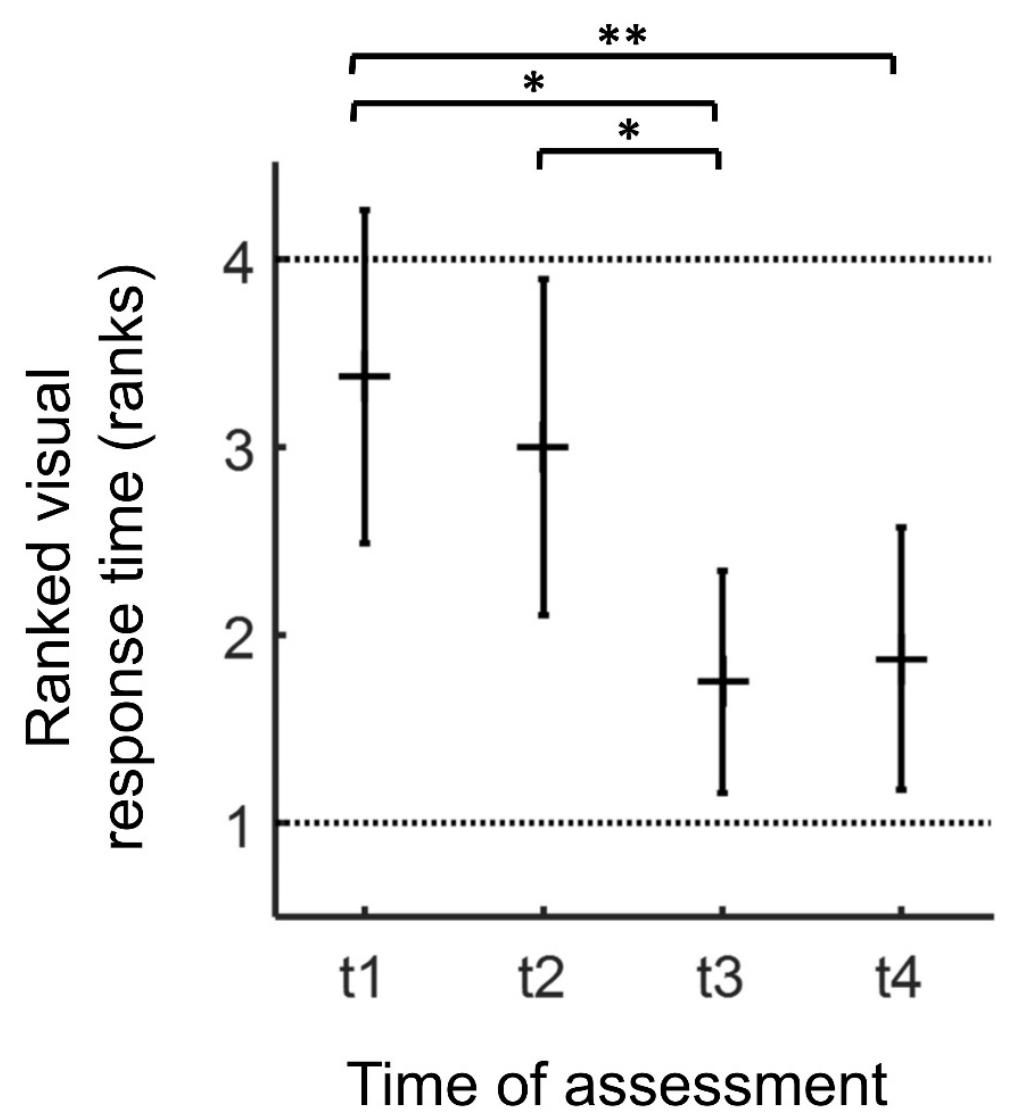

Figure 4: Changes in ranked visual response times during table-top tests of visual search ability before and after training.

Mean ranks of visual response times during table-top tests of visual search ability for the participants as a group where $1=$ fastest visual response time and $4=$ slowest visual response time across all four functional vision assessments. Dotted lines $=$ maximum and minimum ranks, for reference. $*=$ significant difference, $\mathrm{p}<0.05 . * *=$ significant difference, $\mathrm{p}<0.01$. 